\title{
A transference theorem for ergodic $H^{1}$
}

\author{
Nakhlé Asmar and Stephen Montgomery-Smith \\ Department of Mathematics \\ University of Missouri-Columbia \\ Columbia, Missouri 65211 U. S. A.
}

\section{Introduction}

In this paper, we extend the basic transference theorem for convolution operators on $L^{p}$ spaces of Coifman and Weiss [5] to $H^{1}$ spaces. For clarity's sake, we start by recalling the Coifman-Weiss transference theorem for a single convolution operator.

Suppose that $k \in L^{1}(G)$, where $G$ is a locally compact abelian group, and let $N_{p}(k)$ denote the norm of the convolution operator $f \mapsto k * f$, where $f \in L^{p}(G, \lambda)$, and where $\lambda$ is a fixed Haar measure on $G$. Suppose that $R=\left\{R_{u}\right\}_{u \in G}$ is a strongly continuous, uniformly bounded representation of $G$ acting on a general Lebesgue space $L^{p}(\mathcal{M}, \mu)=X_{p}$ where $1 \leq p<\infty$. Let $c_{p}$ be a positive constant such that $\left\|R_{u}\right\| \leq c_{p}$ for all $u \in G$. We use the Bochner integral to define the transferred convolution operator for all $f \in X_{p}$ by

$$
T_{k}(f)=k *_{R} f=\int_{G} R_{-u}(f) k(u) d u,
$$

where here $d u=d \lambda(u)$. It is straightforward to obtain the inequality $\left\|T_{k}(f)\right\|_{L^{p}(\mu)} \leq$ $c_{p}\|k\|_{L^{1}(G)}\|f\|_{L^{p}(\mu)}$. Using the transference methods, one can show that the operator norm of $T_{k}$ does not exceed $c_{p}^{2} N_{p}(k)$. In most cases of interest, when $1<p<\infty, N_{p}(k)$ is much smaller than $\|k\|_{L^{1}(G)}$, and thus there is a clear advantage to the transference methods. By contrast, the case $p=1$ is of little interest since we always have $N_{1}(k)=\|k\|_{L^{1}(G)}$. However, important operators in harmonic analysis are defined on subspaces of $L^{1}(G)$ and have norms smaller than $\|k\|_{L^{1}(G)}$, e.g., singular integral operators on $H^{1}(\mathbf{R})$. One natural question is to ask for the transference of such operators to appropriate subspaces of $L^{1}(\mu)$. When $G=\mathbf{R}$ and the representation $R$ is given by measure-preserving transformations, the subspace of $L^{1}(\mu)$ that is suitable for the transference of operators on $H^{1}(\mathbf{R})$ was introduced by Coifman and Weiss [6], and called ergodic $H^{1}$. The note [6] contains basic properties of ergodic $H^{1}$, derived using sophisticated techniques from [7]. Other interesting properties of ergodic $H^{1}$ are obtained by de la Torre [10], and [2].

Our goal in this paper is to prove a transference theorem for ergodic $H^{1}$. The proofs require new techniques, since the basic averaging process that is behind the methods of [5] does not work when dealing with functions in $H^{1}$. The reader can check that the same proofs apply when $\mathbf{R}$ is replaced by $\mathbf{T}$, the circle group. 
Recently several authors contributed to transference from $H^{1}$. See Liu and Lu [9], and Carro and Soria [4]. These papers have points of contact with our results in the special case of transference from $H^{1}(\mathbf{R})$ to $H^{1}(\mathbf{T})$. We note that the Hardy spaces considered in [4] are different from our transferred spaces, and our main results, Theorems 3.1 and 3.2 below, cannot be derived from any of the cited papers.

The plan of the paper is as follows. In Section 2, we define and study analytic functions in $L^{1}(\mu)$. In Section 3, we prove a transference theorem for maximal operators. In Section 4, we derive some applications along the lines of [6] and [2].

\section{Preliminary results}

Throughout this section, $(\Omega, \mathcal{M}, \mu)$ denotes a measure space, where $\mu$ is an arbitrary measure, $R: u \mapsto R_{u}$ is a uniformly bounded, strongly continuous representation of $\mathbf{R}$ in $L^{1}(\mu)$ such that

$$
\left\|R_{u}\right\| \leq c
$$

We now review a few useful facts from spectral analysis. Let $g \in L^{\infty}(\mathbf{R})$, and let $[g]$ denote the smallest weak-* closed translation invariant subspace of $L^{\infty}(\mathbf{R})$ that contains $g$. The spectrum of $g$, denoted $\operatorname{spec}(g)$, is the set of characters of $\mathbf{R}$ that belong to $[g]$. Define

$$
H^{\infty}(\mathbf{R})=\left\{f \in L^{\infty}(\mathbf{R}): \operatorname{spec}(f) \subset[0, \infty)\right\} .
$$

An equivalent definition of the spectrum is obtained as follows. Let

$$
\mathcal{I}(g)=\left\{f \in L^{1}(\mathbf{R}): f * g=0\right\}
$$

and

$$
Z(\mathcal{I}(g))=\bigcap_{f \in \mathcal{I}(g)}\{s: \widehat{f}(s)=0\}
$$

According to $[8,(40.21)(\mathrm{i})]$, we also have $\operatorname{spec}(g)=Z(\mathcal{I}(g))$. To define the spectrum of a function $f \in L^{1}(\mu)$, let

$$
\mathcal{I}_{R}(f)=\left\{h \in L^{1}(\mathbf{R}): h *_{R} f=0 \mu-\text { a.e. }\right\} .
$$

It is easy to check that $\mathcal{I}_{R}(f)$ is a closed ideal in $L^{1}(\mathbf{R})$.

Definition 2.1 For $f \in L^{1}(\mu)$, define the spectrum of $f$ by

$$
\operatorname{spec}_{R}(f) \equiv Z\left(\mathcal{I}_{R}(f)\right) \equiv \bigcap_{h \in \mathcal{I}_{R}(f)}\{s \in \mathbf{R}: \widehat{h}(s)=0\} .
$$

We say that $f \in L^{1}(\mu)$ is analytic, and write $f \in H^{1}(\mu)$, if $\operatorname{spec}_{R}(f) \subset[0, \infty)$. Hence

$$
H^{1}(\mu)=\left\{f \in L^{1}(\mu): \operatorname{spec}_{R}(f) \subset[0, \infty)\right\} .
$$

The norm in $H^{1}(\mu)$ is the $L^{1}(\mu)$-norm. 
When $\mathbf{R}$ acts on $L^{1}(\mathbf{R}, d x)$ by translation, it is easy to check that the space of analytic functions in $L^{1}(\mathbf{R})$ consists of those functions with Fourier transforms supported in $[0, \infty)$. We set

$$
H^{1}(\mathbf{R})=\left\{f \in L^{1}(\mathbf{R}): \operatorname{supp} \widehat{f} \subset[0, \infty)\right\} .
$$

Let $E$ be a closed subset of $\mathbf{R}$, let

$$
\Im(E)=\left\{f \in L^{1}(\mathbf{R}): \widehat{f}(E) \subset\{0\}\right\},
$$

and let $\mathcal{J}(E)$ denote the closure in $L^{1}(\mathbf{R})$ of the set

$$
\left\{f \in L^{1}(\mathbf{R}): \widehat{f} \text { vanishes on an open set containing } E\right\} \text {. }
$$

Both $\Im(E)$ and $\mathcal{J}(E)$ are closed ideals in $L^{1}(\mathbf{R})$, and we clearly have $\mathcal{J}(E) \subset \Im(E)$. The set $E$ is called a set of spectral synthesis if $\mathcal{J}(E)=\Im(E)$ (see [8, Theorem (40.10)]). The following is a simple consequence of [8, Theorems (40.8), and $(40.10)$ (iii)], and the fact that $[\alpha, \infty)$ and $(-\infty, \alpha]$ are sets of spectral synthesis.

Remark 2.2 Let $\alpha$ be a fixed real number and let $E$ denote the set $[\alpha, \infty)$ or $(-\infty, \alpha]$. Suppose that $g \in L^{\infty}(\mathbf{R})$. Then $\operatorname{spec}(g) \subset E$, if and only if

$$
\int_{\mathbf{R}} f \bar{g} d x=0
$$

for all $f \in \Im(E)$.

Taking $E=[0, \infty)$ in Remark 2.2, we see that a function $g \in L^{\infty}(\mathbf{R})$ belongs to $H^{\infty}(\mathbf{R})$ if and only if

$$
\int_{\mathbf{R}} f g d x=0
$$

for all $f \in H^{1}(\mathbf{R})$.

For $f \in L^{1}(\mu)$ and $A \in \mathcal{M}$, consider the function defined on $\mathbf{R}$ by $t \mapsto \int_{A} R_{t} f d \mu$. It follows easily from the strong continuity and the uniform boundedness of $R$ that this function is in $L^{\infty}(\mathbf{R})$. We now present a useful characterization of $\operatorname{spec}_{R}$.

Proposition 2.3 Let $f \in L^{1}(\mu)$, and let $\alpha$ be any real number. The following are equivalent:

(i) $\operatorname{spec}_{R}(f) \subset[\alpha, \infty)$;

(ii) for every $A \in \mathcal{M}$, the spectrum of the $L^{\infty}(\mathbf{R})$-function $t \mapsto \int_{A} R_{t} f d \mu$ is contained in $[\alpha, \infty)$.

Proof. Let $g \in L^{1}(\mathbf{R})$ be any function in $\mathcal{I}_{R}(f)$, so that $g *_{R} f=0$. Then, for all $t \in \mathbf{R}$, since $g *_{R}\left(R_{t} f\right)=R_{t}\left(g *_{R} f\right)=0$, it follows that, for any $A \in \mathcal{M}$,

$$
\int_{A} g *_{R}\left(R_{t} f\right) d \mu=\int_{\mathbf{R}} g(u) \int_{A} R_{t-u} f d \mu d u=0
$$

From this we see that

$$
g *\left(t \mapsto \int_{A} R_{t} f d \mu\right)=0
$$

which implies that $\mathcal{I}_{R}(f) \subset \mathcal{I}\left(t \mapsto \int_{A} R_{t} f d \mu\right)$. Hence, $Z\left(\mathcal{I}_{R}(f)\right) \supset Z\left(\mathcal{I}\left(t \mapsto \int_{A} R_{t} f d \mu\right)\right)$; equivalently, $\operatorname{spec}_{R}(f) \supset \operatorname{spec}\left(t \mapsto \int_{A} R_{t} f d \mu\right)$. This proves that (i) implies (ii). For 
the other direction, let $E=[\alpha, \infty)$. It is enough to show that $\mathcal{I}_{R}(f) \supseteq \Im(E)$, since this will imply that $\operatorname{spec}_{R}(f) \subset Z(\Im(E))=E$ (see [8, (39.8)(c)]). For this purpose, let $A$ be an arbitrary nonvoid subset in $\mathcal{M}$, and let $g \in \Im(E)$ so that $g^{\sim}$ is also in $\Im(E)$. Applying Remark 2.2, it follows that

$$
\int_{\mathbf{R}} \overline{g(-t)} \overline{\int_{A} R_{t} f d \mu} d t=0
$$

because the spectrum of the function $t \mapsto \int_{A} R_{t} f d \mu$ is contained in $E=[\alpha, \infty)$, and $g^{\sim} \in \Im(E)$. Taking complex conjugates and using Fubini's Theorem, we obtain after changing $t$ to $-t$

$$
\int_{A} \int_{\mathbf{R}} g(t) R_{-t} f d t d \mu=0
$$

Since this holds for all $A \in \mathcal{M}$, we conclude that $\int_{\mathbf{R}} g(t) R_{-t} f d t=0 \mu$-a. e. which is what we want to prove.

The following is a useful characterization of $H^{1}(\mu)$.

Proposition 2.4 Let $f \in L^{1}(\mu)$. The following are equivalent:

(i) $f \in H^{1}(\mu)$;

(ii) for every $A \in \mathcal{M}$, the function $t \mapsto \int_{A} R_{t} f d \mu$ is in $H^{\infty}(\mathbf{R})$;

(iii) for every $A \in \mathcal{M}$ and every $g \in H^{1}(\mathbf{R})$, we have

$$
\int_{\mathbf{R}} g(t) \int_{A} R_{t} f d \mu d t=0
$$

(iv) for every $h \in \Im([0, \infty))$, we have $h *_{R} f=0 \mu$-a.e.

Proof. The equivalence $(\mathrm{i}) \Leftrightarrow$ (ii) follows from Proposition 2.3 and definitions. The equivalence (ii) $\Leftrightarrow$ (iii) follows from (4). To prove (iii) $\Rightarrow($ iv), let $h \in \Im([0, \infty))$. Since the function $t \mapsto h(-t)$ is in $H^{1}(\mathbf{R})$, it follows from (iii) that

$$
\int_{\mathbf{R}} h(-t) \int_{A} R_{t} f d \mu d t=0
$$

for all $A \in \mathcal{M}$; equivalently,

$$
\int_{\mathbf{R}} h(t) \int_{A} R_{-t} f d \mu d t=\int_{A} \int_{\mathbf{R}} h(t) R_{-t} f d t d \mu=0,
$$

for all $A \in \mathcal{M}$, which implies (iv). The proof of (iv) $\Rightarrow$ (iii) is simple and will be omitted.

The following simple proposition is very useful.

Proposition 2.5 Suppose that $f, f_{n} \in L^{1}(\mu)$, and $k \in L^{1}(\mathbf{R})$. Then

(i) $\operatorname{spec}_{R}\left(k *_{R} f\right) \subset \operatorname{supp} \widehat{k} \cap \operatorname{spec}_{R}(f)$.

(ii) Suppose that $\operatorname{spec}_{R}\left(f_{n}\right) \subset[\alpha, \infty)$ and $f_{n} \rightarrow f$ in $L^{1}(\mu)$, then $\operatorname{spec}_{R}(f) \subset[\alpha, \infty)$.

The proof of (i) is simple and will be omitted. For the proof of (ii), use Proposition 2.3(ii) and Dominated Convergence.

For use in the sequel, we introduce the space $H^{1}\left(\mathbf{R}, L^{1}(\mu)\right)$ which consists of Bochner integrable functions $g$ on $\mathbf{R}$ with values in $L^{1}(\mu)$ such that

$$
\int_{\mathbf{R}} g(x) e^{-i x t} d x=0, \text { for all } t<0 .
$$

From definitions, a function $g \in H^{1}\left(\mathbf{R}, L^{1}(\mu)\right)$ is jointly measurable on $\mathbf{R} \times \Omega$ and belongs to $L^{1}(\mathbf{R} \times \Omega, d x d \omega)$. 
Lemma 2.6 A function $g$ is in $H^{1}\left(\mathbf{R}, L^{1}(\mu)\right)$ if and only if for $\mu$-almost all $\omega \in \Omega$ the mapping $x \mapsto g(x, \omega)$ is in $H^{1}(\mathbf{R})$.

Proof. One direction is clear: if for almost all $\omega \in \Omega$ the mapping $x \mapsto g(x, \omega)$ is in $H^{1}(\mathbf{R})$, then (5) holds and so $g \in H^{1}\left(\mathbf{R}, L^{1}(\mu)\right)$. Now suppose that $g \in H^{1}\left(\mathbf{R}, L^{1}(\mu)\right)$. Because of (5), for each $t<0$, there is a subset $B_{t} \subset \Omega$ such that $\mu\left(\Omega \backslash B_{t}\right)=0$, and, for all $\omega \in B_{t}$, we have

$$
\int_{\mathbf{R}} g(x, \omega) e^{-i x t} d x=0 .
$$

Let $\left(t_{n}\right)=\mathbf{Q} \cap(-\infty, 0)$ denote the set of negative rational numbers, and let $B=$ $\bigcap_{n} B_{t_{n}}$. Then $\mu(\Omega \backslash B)=0$, and (6) holds for all $\omega \in B$ and $\left(t_{n}\right)$. Now, given an arbitrary real number $t<0$, choose a subsequence $\left(t_{n_{j}}\right)$ from $\left(t_{n}\right)$ such that $t_{n_{j}} \rightarrow t$. Then, for $\omega \in B$, we have $g(x, \omega) e^{-i x t_{n_{j}}} \rightarrow g(x, \omega) e^{-i x t}$ for all $x \in \mathbf{R}$. Hence, by Dominated Convergence, we have, for all $\omega \in B$,

$$
0=\int_{\mathbf{R}} g(x, \omega) e^{-i x t_{n_{j}}} d x \rightarrow \int_{\mathbf{R}} g(x, \omega) e^{-i x t} d x,
$$

implying (5).

Using the representation $R$, for each $\alpha \in \mathbf{R}$, we define a new representation $e^{i \alpha(\cdot)} R$ by: $u \in \mathbf{R} \mapsto e^{i \alpha u} R_{u}$. The following simple properties will be very useful.

Lemma 2.7 Suppose that $f \in H^{1}(\mu)$ and $k \in L^{1}(\mathbf{R})$, and let $\alpha \geq 0$. Then

(i) $\operatorname{spec}_{e^{i \alpha(\cdot)} R}(f) \subset[\alpha, \infty)$;

(ii) $\lim _{\alpha \rightarrow 0}\left\|k *_{e^{i \alpha(\cdot)} R} f-k *_{R} f\right\|_{L^{1}(\mu)}=0$, where here $\alpha \rightarrow 0$ through a countable sequence.

Proof. To prove (i), it is enough to show that for any $s_{0}<\alpha$, we can find a function $h \in L^{1}(\mathbf{R})$ such that $h *_{e^{i \alpha(\cdot)}} f=0$ and $\widehat{h}\left(s_{0}\right) \neq 0$. Since $\left(s_{0}-\alpha\right) \notin \operatorname{spec}_{R}(f) \subset[0, \infty)$, we can find a function $g \in L^{1}(\mathbf{R})$ such that $g *_{R} f=0$ and $\widehat{g}\left(s_{0}-\alpha\right)=1$. We clearly have

$$
\left(e^{i \alpha(\cdot)} g\right) *_{e^{i \alpha(\cdot)}} f=g *_{R} f=0,
$$

and since $\left(\widehat{e^{i \alpha(\cdot)}} g\right)\left(s_{0}\right)=\widehat{g}\left(s_{0}-\alpha\right)=1$, the proof of (i) is complete. For (ii), we have

$$
\begin{aligned}
\left\|k *_{e^{i \alpha(\cdot) R}} f-k *_{R} f\right\|_{L^{1}(\mu)} & =\int_{\Omega}\left|\int_{\mathbf{R}}\left(e^{-i \alpha u}-1\right) R_{-u} f k(u) d u\right| d|\mu| \\
& \leq \int_{\mathbf{R}} \int_{\Omega}\left|R_{-u} f\right| d|\mu|\left|e^{-i \alpha u}-1\right||k(u)| d u \\
& \leq c\|f\|_{L^{1}(\mu)} \int_{\mathbf{R}}\left|e^{-i \alpha u}-1\right||k(u)| d u .
\end{aligned}
$$

Now (ii) follows from the fact that $\lim _{\alpha \rightarrow 0} 1-e^{-i \alpha u}=0$ for all $u$, and Dominated Convergence.

\section{Transference of maximal inequalities}

Throughout this section, $(\Omega, \mathcal{M}, \mu)$ is a measure space where $\mu$ is an arbitrary measure. Given $k \in L^{1}(\mathbf{R})$, we let $N(k)$ denote the norm of the convolution operator $f \mapsto k * f$ from $H^{1}(\mathbf{R})$ into $H^{1}(\mathbf{R})$. All other notation is as in the previous section. Our transference theorem for a single convolution operator follows. 
Theorem 3.1 Let $R$ be a strongly continuous uniformly bounded representation of $\mathbf{R}$ acting on $L^{1}(\mu)$ such that $\left\|R_{u}\right\| \leq c$ for all $u \in \mathbf{R}$, where $c$ is a positive constant. Let $k \in L^{1}(\mathbf{R})$. For all $f \in H^{1}(\mu)$ we have

$$
\left\|k *_{R} f\right\|_{L^{1}(\mu)} \leq c^{2} N(k)\|f\|_{L^{1}(\mu)} .
$$

Under appropriate additional conditions on $R$, this result can be extended to maximal operators corresponding to sequences of convolution operators. For later applications we will state and prove the more general version for maximal operators. A few more definitions are needed. (For background and references, see [1].)

A linear mapping $T$ of $L^{1}(\mu)$ is called separation-preserving (respectively, positivitypreserving) if whenever $f \in L^{1}(\mu), g \in L^{1}(\mu)$, and $f g=0 \mu-a$.e., on $\Omega$, (respectively, $f \geq 0, \mu-a$. e.), then $(T f)(T g)=0 \mu$ a. e. on $\Omega$ (respectively, $T f \geq 0, \mu-a$.e.). If $T$ is separation-preserving, then there is a positivity-preserving operator $|T|$ such that for all $f \in L^{1}(\mu)$, we have $|T f|=|T|(|f|), \mu-a$. e.

Let $\left\{k_{n}\right\} \subset L^{1}(\mathbf{R})$ and denote by $N\left(\left\{k_{n}\right\}\right)$ the smallest constant such that

$$
\left\|\sup _{n \geq 1}\left|k_{n} * f\right|\right\|_{L^{1}(\mathbf{R})} \leq N\left(\left\{k_{n}\right\}\right)\|f\|_{L^{1}(\mathbf{R})},
$$

for $f \in H^{1}(\mathbf{R})$.

Theorem 3.2 Suppose that $R$ is a strongly continuous, uniformly bounded representation of $\mathbf{R}$ in $L^{1}(\mu)$ by separation-preserving operators. Then for all $f \in H^{1}(\mu)$, we have

$$
\left\|\sup _{n \geq 1}\left|k_{n} *_{R} f\right|\right\|_{L^{1}(\mu)} \leq c^{2} N\left(\left\{k_{n}\right\}\right)\|f\|_{L^{1}(\mu)} .
$$

The proof of this theorem will be done in several steps. The reader can check that separation-preserving is only needed for the transference of maximal inequalities, and so the proof that we present applies also to Theorem 3.1.

We start with a simple transference result to a space of vector-valued functions.

Lemma 3.3 Suppose that $g$ is a function in $H^{1}\left(\mathbf{R}, L^{1}(\mu)\right)$. Then,

$$
\int_{\mathbf{R}}\left\|\max _{1 \leq j \leq N}\left|\int_{\mathbf{R}} g(x-t) k_{j}(t) d t\right|\right\|_{L^{1}(\mu)} d x \leq N\left(\left\{k_{j}\right\}\right)\|g\|_{L^{1}\left(\mathbf{R}, L^{1}(\mu)\right)} .
$$

Proof. Using Fubini's Theorem and the fact that $x \mapsto g(x, \omega)$ is in $H^{1}(\mathbf{R})$ for $\mu$-almost all $\omega$, we get

$$
\begin{aligned}
\int_{\Omega} \int_{\mathbf{R}} \max _{1 \leq j \leq N}\left|\int_{\mathbf{R}} g(x-t, \omega) k_{j}(t) d t\right| d x d \mu & \leq N\left(\left\{k_{j}\right\}\right) \int_{\Omega} \int_{\mathbf{R}}|g(x, \omega)| d x d \mu \\
& =N\left(\left\{k_{j}\right\}\right)\|g\|_{L^{1}\left(\mathbf{R}, L^{1}(\mu)\right)}
\end{aligned}
$$

which is what we want. 
Lemma 3.4 Let $\epsilon>0$, and suppose that $g \in L^{1}(\mathbf{R})$ has the following properties:

(i) $g \geq 0$,

(ii) $\widehat{g}(0)=\int_{\mathbf{R}} g d x=1$,

(iii) $\widehat{g}$ has compact support contained in $\left(\frac{-\epsilon}{2}, \frac{\epsilon}{2}\right)$.

Then for any function $f \in H^{1}(\mu)$ with $\operatorname{spec}_{R}(f) \subset[\epsilon, \infty)$, we have that the function $u \mapsto g(u) R_{u} f$ is in $H^{1}\left(\mathbf{R}, L^{1}(\mu)\right)$.

Proof. We need to check that for any $s<0$

$$
\int_{\mathbf{R}} e^{-i s u} g(u) R_{u} f d u=0 \mu-a . e .
$$

This will follow if we can show that for any $A \in \mathcal{M}$ we have

$$
\int_{A} \int_{\mathbf{R}} e^{-i s u} g(u) R_{u} f d u d \mu=\int_{\mathbf{R}} e^{-i s u} g(u) \int_{A} R_{u} f d \mu d u=0 .
$$

Equivalently, by taking complex conjugates, it suffices to show that

$$
\int_{\mathbf{R}} e^{i s u} g(u) \overline{\int_{A} R_{u} f d \mu} d u=0
$$

By Proposition 2.3, the spectrum of the the function $u \mapsto \int_{A} R_{u} f d \mu$ is contained in $[\epsilon, \infty)$. Since the support of the Fourier transform of the function $u \mapsto e^{i s u} g(u)$ is contained in $\left(-\frac{\epsilon}{4}+s, \frac{\epsilon}{4}+s\right)$, and $s \leq 0$, we have that $e^{i s u} g(u) \in \Im([\epsilon, \infty))$, and $(9)$ follows from Remark 2.2.

The proof of (7) will be facilitated by the following two reductions.

First reduction In proving (7), it is enough to assume that the sequence $\left\{k_{n}\right\}$ is finite. This is a simple consequence of Monotone Convergence.

Henceforth, we assume that $n$ ranges from 1 to $N$, where $N$ is a fixed positive integer and, instead of (7), prove the inequality

$$
\left\|\max _{1 \leq n \leq N}\left|k_{n} *_{R} f\right|\right\|_{L^{1}(\mu)} \leq c^{2} N\left(\left\{k_{n}\right\}\right)\|f\|_{L^{1}(\mu)},
$$

for all $f \in H^{1}(\mu)$.

Second reduction In proving (10), it is enough to consider functions $f \in H^{1}(\mu)$ with $\operatorname{spec}_{R}(f) \subset[\epsilon, \infty)$, where $\epsilon>0$.

To justify this reduction, suppose that (10) holds whenever a representation $R$ is separation-preserving, strongly continuous, uniformly bounded with constant $c$, and $f$ has its spectrum contained in $[\epsilon, \infty)$ where $\epsilon>0$. Let $\alpha>0$, and consider the representation $e^{i \alpha(\cdot)} R$. It is clear that this representation enjoys all the stated properties of $R$ (strong continuity, uniform boundedness with the same constant $c$, and separation-preserving). Moreover, if $f \in H^{1}(\mu)$, then $\operatorname{spec}_{e^{i \alpha(\cdot)} R} f \subset[\alpha, \infty)$, by Lemma 2.7(i). Hence, by our assumption,

$$
\left\|\max _{1 \leq n \leq N}\left|k_{n} *_{e^{i \alpha(\cdot)} R} f\right|\right\|_{L^{1}(\mu)} \leq c^{2} N\left(\left\{k_{n}\right\}\right)\|f\|_{L^{1}(\mu)} .
$$

Letting $\alpha \downarrow 0$, and using Lemma 2.7, we have that, for each $n \in\{1,2, \ldots, N\}$, $k_{n} *_{e^{i \alpha(\cdot)} R} f \rightarrow k_{n} *_{R} f$ in $L^{1}(\mu)$. From this and (11), the inequality (10) follows easily, establishing the second reduction. 
Proof of Theorem 3.2 Suppose that $f \in L^{1}(\mu)$ with $\operatorname{spec}_{R}(f) \subset[\epsilon, \infty)$ where $\epsilon$ is a fixed positive number. Let $g$ be as in Lemma 3.4 and let $F(t)=g(t) R_{t} f$. By Lemma 3.4, $F \in H^{1}\left(\mathbf{R}, L^{1}(\mu)\right)$, and so, by Lemma 3.3, we have

$$
\int_{\mathbf{R}}\left\|\max _{1 \leq n \leq N}\left|\int_{\mathbf{R}} F(x-t) k_{n}(t) d t\right|\right\|_{L^{1}(\mu)} d x \leq N\left(\left\{k_{n}\right\}\right)\|F\|_{L^{1}\left(\mathbf{R}, L^{1}(\mu)\right)} .
$$

We now proceed to show that (10) is a consequence of (12). We have

$$
\begin{aligned}
\|F\|_{L^{1}\left(\mathbf{R}, L^{1}(\mu)\right)} & =\int_{\mathbf{R}} \int_{\Omega}\left|g(t) R_{t} f\right| d|\mu| d t \\
& =\int_{\mathbf{R}} g(t) \int_{\Omega}\left|R_{t} f\right| d|\mu| d t \leq c\|f\|_{L^{1}(\mu)} .
\end{aligned}
$$

Using the fact that $R$ is a uniformly bounded and strongly continuous representation by separation-preserving operators, we obtain

$$
\begin{aligned}
\max _{1 \leq n \leq N}\left|\int_{\mathbf{R}} F(x-t) k_{n}(t) d t\right| & =\max _{1 \leq n \leq N}\left|\int_{\mathbf{R}}\left(R_{x-t} f\right) g(x-t) k_{n}(t) d t\right| \\
& =\max _{1 \leq n \leq N}\left|R_{x}\right|\left(\left|\int_{\mathbf{R}}\left(R_{-t} f\right) g(x-t) k_{n}(t) d t\right|\right) .
\end{aligned}
$$

Since $\left|R_{ \pm x}\right|$ is positivity-preserving and since $\left|R_{x}\right|^{-1}=\left|R_{-x}\right|$, we obtain after applying $\left|R_{-x}\right|$ to both sides of the last equality

$$
\left|R_{-x}\right|\left(\max _{1 \leq n \leq N}\left|\int_{\mathbf{R}} F(x-t) k_{n}(t) d t\right|\right) \geq \max _{1 \leq n \leq N}\left|\int_{\mathbf{R}}\left(R_{-t} f\right) g(x-t) k_{n}(t) d t\right| .
$$

Hence, using the last inequality and the uniform boundedness of $R$, we obtain

$$
c\left\|\max _{1 \leq n \leq N}\left|\int_{\mathbf{R}} F(x-t) k_{n}(t) d t\right|\right\|_{L^{1}(\mu)} \geq\left\|\max _{1 \leq n \leq N}\left|\int_{\mathbf{R}}\left(R_{-t} f\right) g(x-t) k_{n}(t) d t\right|\right\|_{L^{1}(\mu)} .
$$

Integrating both sides of (14) over $\mathbf{R}$ in the $x$ variable, and using (12) and (13), we obtain

$$
N\left(\left\{k_{n}\right\}\right) c^{2}\|f\|_{L^{1}(\mu)} \geq \int_{\mathbf{R}} \int_{\Omega} \max _{1 \leq n \leq N}\left|\int_{\mathbf{R}}\left(R_{-t} f\right) g(x-t) k_{n}(t) d t\right| d|\mu| d x .
$$

Obvious manipulations with (15), Fubini's Theorem and the fact that $g \geq 0$ and $\widehat{g}(0)=\int_{\mathbf{R}} g=1$, yield

$$
\begin{aligned}
N\left(\left\{k_{n}\right\}\right) c^{2}\|f\|_{L^{1}(\mu)} & \geq \int_{\Omega} \int_{\mathbf{R}} \max _{1 \leq n \leq N}\left|\int_{\mathbf{R}}\left(R_{-t} f\right) g(x-t) k_{n}(t) d t\right| d x d|\mu| \\
& \geq \int_{\Omega} \max _{1 \leq n \leq N}\left|\int_{\mathbf{R}} g(x-t) d x \int_{\mathbf{R}}\left(R_{-t} f\right) k_{n}(t) d t\right| d|\mu| \\
& =\int_{\Omega} \max _{1 \leq n \leq N}\left|\int_{\mathbf{R}}\left(R_{-t} f\right) k_{n}(t) d t\right| d|\mu| \\
& =\left\|\max _{1 \leq n \leq N}\left|k_{n} *_{R} f\right|\right\|_{L^{1}(\mu)},
\end{aligned}
$$

which proves (10). 


\section{$4 \quad H^{1}(\mu)$ and the ergodic Hilbert transform}

In this section we will investigate a connection between $H^{1}(\mu)$, the space ergodic $H^{1}$ of [6], and spaces of functions introduced in [2] (Theorem 4.1 below). Throughout, $u \rightarrow R_{u}$ will denote a strongly continuous representation of $\mathbf{R}$ by measure-preserving transformations on an finite measure space $(\Omega, \mathcal{M}, \mu)$. In particular, $R$ is separationpreserving and uniformly bounded with $c=1$. (The results of this section apply as well in the more general setting of distributionally controlled representations that were introduced in [2]. For clarity's sake, we will only discuss representations given by measure-preserving transformations.) Since the measure $\mu$ is finite, we have the following useful direct sum decomposition of $L^{1}(\mu)$ :

$$
L^{1}(\mu)=Y \bigoplus Z
$$

where

$$
Y=\left\{f \in L^{1}(\mu): R_{u} f=f, \text { for all } u \in \mathbf{R}\right\},
$$

and $Z$ is the $L^{1}(\mu)$-closure of the linear subspace of $L^{1}(\mu)$ spanned by the ranges of the operators $f \mapsto g *_{R} f$, for all $g \in L^{1}(\mathbf{R})$ such that $\mathbf{R} \backslash\{0\}$ contains the support of $\hat{g}$. (See [2, Proposition (3.19)].)

Let $h(t)=\frac{1}{\pi t}$ for $t \neq 0$ denote the Hilbert kernel. For each $n$, let $h_{n}$ denote the $n$th truncate $h_{n}(t)=\frac{1}{\pi t}$ if $\frac{1}{n}<|t|<n$, and $h_{n}(t)=0$ otherwise. For $f \in L^{1}(\mathbf{R})$, denote its Hilbert transform by $\widetilde{f}$. It is a classical fact that if $f \in H^{1}(\mathbf{R})$, then $h_{n} * f \rightarrow \tilde{f}$ in $L^{1}(\mathbf{R})$, and in this case, we have $\tilde{f}=-i f$. (Recall that $H^{1}(\mathbf{R})$ consists of all functions in $L^{1}(\mathbf{R})$ with Fourier transform vanishing on $(-\infty, 0]$.) Since the space $H^{1}(\mathbf{R})$ is a Banach space with the $L^{1}(\mathbf{R})$ norm, it follows from the Uniform Boundedness Principle that there is a positive constant $C$ such that for all $n$ and all $f \in H^{1}(\mathbf{R})$

$$
\left\|h_{n} * f\right\|_{1} \leq C\|f\|_{1} .
$$

For $f \in L^{1}(\mu)$, we define the ergodic Hilbert transform of $f$ by

$$
\mathcal{H} f=\lim _{n} h_{n} *_{R} f \mu \text { a.e.. }
$$

It is a well-known consequence of the transference methods that the limit exists $\mu$-a.e. and that the operator $f \mapsto \mathcal{H} f$ is of weak type $(1,1)$ with weak type norm smaller than the weak type $(1,1)$ norm of the Hilbert transform. Also, the maximal operator $f \mapsto \sup _{n}\left|h_{n} *_{R} f\right|$ is of weak type $(1,1)$ with weak type norm smaller than the weak type $(1,1)$ norm of the maximal Hilbert transform on $L^{1}(\mathbf{R})$. (See [3, 5], or [2] for the case of distributionally controlled representations.)

Following Coifman and Weiss [6], we define the space ergodic $H^{1}$ as the class of all functions of the form $f+i \mathcal{H} f \in L^{1}(\mu)$. We also recall form [2], Section 3, the space

$$
\mathcal{A}(R)=\left\{f \in L^{1}(\mu): \mathcal{H} f \in L^{1}(\mu)\right\} .
$$

A connection between $H^{1}(\mu)$ and ergodic $H^{1}$ is described by the following theorem.

Theorem 4.1 Suppose that $f \in L^{1}(\mu)$. Then,

(i) $f \in H^{1}(\mu) \Rightarrow f \in \mathcal{A}(R)$ and $\|\mathcal{H} f\|_{1} \leq C\|f\|_{1}$ where $C$ is as in (16);

(ii) $f \in \mathcal{A}(R) \Rightarrow f+i \mathcal{H} f \in H^{1}(\mu)$. 
We present the proof in a sequence of steps.

Proposition 4.2 If $f \in H^{1}(\mu)$, then

$$
\sup _{n}\left\|h_{n} *_{R} f\right\|_{L^{1}(\mu)} \leq C\|f\|_{L^{1}(\mu)},
$$

where $C$ is as in (16).

Proof. As a consequence of Proposition 2.5(ii), we have that $H^{1}(\mu)$ is a Banach space with the $L^{1}(\mu)$-norm. The desired result is now a simple consequence of the Uniform Boundedness Principle, Theorem 3.1, and (16).

Proof of Theorem 4.1(i). Let $f \in H^{1}(\mu)$. We have $h_{n} *_{R} f \rightarrow \mathcal{H} f \mu$-a.e. Applying Proposition 4.2 and Fatou's Lemma, we get the desired conclusions.

Toward the proof of Theorem 4.1(ii), we present the following result.

Proposition 4.3 Suppose that $f \in H^{1}(\mu)$, and write $f=f_{1}+f_{2}$, where $f_{1} \in Y$ and $f_{2} \in Z$, then $f_{2} \in H^{1}(\mu)$.

Proof. According to Proposition 2.4(iv), it is enough to show that for every $h \in \Im([0, \infty))$ we have $h *_{R} f_{2}=0$. Note that for $h \in \Im([0, \infty))$, we have $\int_{\mathbf{R}} h d x=$ $\widehat{h}(0)=0$. Now, $h *_{R} f_{2}=h *_{R} f-h *_{R} f_{1}=h *_{R} f-f_{1} \int_{\mathbf{R}} h d x=h *_{R} f$. Since $f \in H^{1}(\mu)$, we have by Proposition 2.4(iv) $h *_{R} f=0$ and so $h *_{R} f_{2}=0$.

Before we return to the proof of Theorem 4.1 we recall certain properties from [2].

Remark 4.4 (i) Suppose that $f \in \mathcal{A}(R)$, then $h_{n} *_{R} f \rightarrow \mathcal{H} f$ in $L^{1}(\mu)$ (see [2, Theorem (3.24)]).

(ii) Following [2, Definition (3.29)], let

$$
H^{1}(R)=\left\{f \in L^{1}(\mu): f=i \mathcal{H} f\right\} .
$$

Then, according to [2, Theorem (3.34)], we have

$$
H^{1}(R)=\left\{f+i \mathcal{H} f: f \in Z, \text { and } \mathcal{H} f \in L^{1}(\mu)\right\}
$$

(iii) Using (i), we see that if $f \in H^{1}(R)$, then $f \in \mathcal{A}(R)$ and hence, $\mu$-a. e. , we have

$$
f=i \mathcal{H} f=i \lim _{n} h_{n} *_{R} f \text {. }
$$

Proof of Theorem 4.1(ii). We first show that $H^{1}(R) \subset H^{1}(\mu)$. Let $f \in$ $H^{1}(R)$. By Proposition 2.4, it is enough to show that for every $h \in \Im([0, \infty))$ we have $h *_{R} f=0$. For $h \in \Im([0, \infty))$, we easily see that $\widetilde{h} \in L^{1}(\mathbf{R})$ and $\widetilde{h}=i h$. Hence, $h_{n} * h \rightarrow \widetilde{h}=i h$ in $L^{1}(\mathbf{R})$. Now, using Remark 4.4(iii) and convergence in $L^{1}(\mu)$, we can write

$$
\begin{aligned}
h *_{R} f & =h *_{R}(i \mathcal{H} f) \\
& =i h *_{R}\left(\lim _{n} h_{n} *_{R} f\right) \\
& =i \lim _{n}\left(h * h_{n}\right) *_{R} f=-h *_{R} f,
\end{aligned}
$$


implying that $h *_{R} f=0 \mu$-a.e., and so $H^{1}(R) \subset H^{1}(\mu)$.

Now suppose that $f \in \mathcal{A}(R)$ and write $f=f_{1}+f_{2}$ with $f_{1} \in Y, f_{2} \in Z$. We have $f+i \mathcal{H} f=f_{1}+f_{2}+i \mathcal{H} f_{2}$. We have $f_{2}+i \mathcal{H} f_{2} \in H(R) \subset H^{1}(\mu)$. Also, we trivially have $f_{1} \in H^{1}(\mu)$. Hence it follows that $f+i \mathcal{H} f \in H^{1}(\mu)$.

Ergodic $H^{1}$ We end the paper by mentioning how Theorem 3.2 can be used to simplify some of the proofs in the maximal characterization of ergodic $H^{1}$ in [6]. As is done in [6], we introduce a maximal convolution operator $M$ that characterizes $H^{1}(\mathbf{R})$ and such that the kernels of the convolution operators have compact support. This operator can be defined by the dilates of a single smooth function with compact support (see [6, Section 1]). For real-valued functions $f \in L^{1}(\mathbf{R})$, we have

$$
c_{1}\|M f\|_{1} \leq\|f\|_{1}+\|\tilde{f}\|_{1} \leq c_{2}\|M f\|_{1},
$$

where $c_{1}$ and $c_{2}$ are absolute constants. Let $M^{\sharp}$ denote the transferred maximal operator defined on $L^{1}(\mu)$. Coifman and Weiss [6] proved that there are positive constants $c_{1}$ and $c_{2}$ such that for all real-valued $f \in L^{1}(\mu)$, we have

$$
c_{1}\left\|M^{\sharp} f\right\|_{1} \leq\|f\|_{1}+\|\mathcal{H} f\|_{1} \leq c_{2}\left\|M^{\sharp} f\right\|_{1} .
$$

The second inequality in (18) follows directly using the transference methods, as shown in [6], following Lemma 2.7. The proof of the first inequality in (18) as presented in [6] is much more involved. We will show here that this inequality is a simple consequence of Theorem 3.2 and the first inequality in (17). Indeed, suppose that $f$ is a real-valued function in $L^{1}(\mu)$ and $\mathcal{H} f$ is also in $L^{1}(\mu)$. Then, by Theorem 4.1, $f+i \mathcal{H} f \in H^{1}(\mu)$. Note that for $f \in H^{1}(\mathbf{R})$, the first inequality in (17) states that

$$
c_{1}\|M f\|_{1} \leq 2\|f\|_{1}
$$

Applying Theorem 3.2 with $f+i \mathcal{H} f \in H^{1}(\mu)$, and using (19) and Theorem 4.1(i), we get

$$
\left\|M^{\sharp} f\right\|_{1} \leq\left\|M^{\sharp}(f+i \mathcal{H} f)\right\|_{1} \leq 2 c_{1}^{-1}\|f+i \mathcal{H} f\|_{1} \leq 2 c_{1}^{-1}(1+C)\|f\|_{1} .
$$

Acknowledgements The work of the authors was supported by separate grants from the National Science Foundation (U.S.A.).

\section{References}

[1] N. Asmar, E. Berkson, and T. A. Gillespie, Transference of strong type maximal inequalities by separation-preserving representation, Amer. J. Math. 113 (1991), 47-74.

[2] N. Asmar, E. Berkson, and T. A. Gillespie, Distributional control and generalized analyticity, Integral Equations and Operator Theory 14 (1991), 311-341.

[3] A. Calderón, Ergodic theory and translation-invariant operators, Proc. Nat. Acad. Sci. , 157 (1971), 137-153. 
[4] M. J. Carro and J. Soria, Transference theory on Hardy and Sobolev spaces, preprint.

[5] R. R. Coifman and G. Weiss, "Transference methods in analysis", Regional Conference Series in Math. 31, Amer. Math. Soc., Providence, R. I. 1977.

[6] R. R. Coifman and G. Weiss, Maximal functions on $H^{p}$ spaces defined by ergodic transformations, Proc. Nat. Acad. Sci. , (U. S. A.) 70 (1973), 17611763.

[7] C. Fefferman, and E. Stein $H^{p}$-spaces in several variables, Acta Math, 129, (1972), 137-193.

[8] E. Hewitt and K. A. Ross, "Abstract Harmonic Analysis II," Grundlehren der Math. Wissenschaften in Einzeldarstellungen, Band 152, SpringerVerlag, New York, 1970.

[9] Z. Liu and S. Lu, Transference and restriction of maximal multiplier operators on Hardy spaces, Studia Math. 105 (1993), 121-134.

[10] A. de la Torre, Ergodic $H^{1}$, Bol. Soc. Mat. Mexicana, 22 (1977), 10-22. 